\title{
UMA ABORDAGEM DIDÁTICA PARA O ESTUDO DE AMPLIFICADORES ELETRÔNICOS
}

\author{
A DIDACTIC APPROACH FOR THE STUDY OF ELECTRONIC AMPLIFIERS
}

Francisco Antônio Tadheu Ramos ${ }^{1}$, Francisco José Arnold ${ }^{2}$

DOI: 10.37702/REE2236-0158.v40p407-417.2021

\begin{abstract}
RESUMO
A eletrônica analógica é um tema mandatório nas grades curriculares de diversos cursos de engenharia. As características dessa disciplina facilitam a proposição de trabalhos que integrem conhecimentos de outras áreas, o que é desejável neste momento de inserção de novas metodologias de ensino. Este trabalho propõe uma atividade de estudo do desempenho de amplificadores classe B que acionam um transdutor piezelétrico. As variações das propriedades físicas do transdutor modificam a carga do amplificador. Essas modificações introduzem componentes reativas que afetam o balanço de potências no amplificador. Os resultados mostram que a fase do sinal de saída produz diminuição de eficiência e aumento da potência dissipadas nos transistores. A abordagem ao tema feita neste trabalho não é encontrada nos livros didáticos de eletrônica analógica e pode ser empregada em atividades conjuntas de disciplinas nos cursos de engenharia.
\end{abstract}

Palavras-chave: classe B; circuitos equivalentes; transdutores; piezelétrico.

\begin{abstract}
Analog electronics is a mandatory theme in the curriculum of many engineering courses. The characteristics of this discipline facilitate the proposition of works that integrate knowledge from other areas, which is desirable at this time of insertion of new teaching methodologies. This work proposes an activity to study the performance of class B amplifiers that drive a piezoelectric transducer. Variations in the transducer's physical properties change the amplifier load. These modifications introduce reactive components that affect the power balance in the amplifier. The results show that the phase of the output signal produces a decrease in efficiency and an increase in power dissipated in the transistors. The approach to the subject made in this work is not found in textbooks on analog electronics and can be used in joint activities of disciplines in engineering courses.
\end{abstract}

Keywords: Class-B; equivalent circuits; transducers; piezoelectric.

\section{INTRODUÇÃO}

Os cursos de graduação em engenharia com foco na área de eletricidade, especialmente nas modalidades elétrica, telecomunicações e mecatrônica possuem em suas grades curriculares a disciplina Eletrônica analógica. Trata-se de uma disciplina que possui conteúdo básico para a compreensão do funcionamento de componentes eletrônicos e para o desenvolvimento de circuitos e sistemas que são explorados no escopo desses cursos. No

\footnotetext{
${ }^{1}$ Engenheiro Eletricista, Instituto Superior de Ciências Aplicadas, Limeira - SP, tadheuramos@yahoo.com.br

${ }^{2}$ Prof. Dr. em Engenharia, Universidade Estadual de Campinas, Limeira - SP, arnold@ unicamp.br
} 
passado o ensino de eletrônica analógica era baseado no oferecimento de aulas teóricas e práticas de laboratório nas quais o aluno podia construir circuitos e realizar medições para se aprofundar na aplicação específica do tema estudado. Nas últimas duas décadas o uso de softwares de simulação foi incentivado e disseminado, trazendo com isso não apenas uma ferramenta para desenvolvimento de projetos, mas também para apoio às atividades de ensino.

Mais recentemente, também em razão da implantação das novas diretrizes curriculares para os cursos de engenharia (DIRETRIZES CURRICULARES NACIONAIS, 2019), novas metodologias de ensino estão sendo introduzidas e incentivadas. Um dos aspectos enfatizados nas diretrizes curriculares é a busca pela integração de saberes que pode ser promovida pela realização de trabalhos e projetos que reúnam conteúdos de diferentes disciplinas. Este trabalho apresenta uma proposta de atividade que pode ser aplicada nas aulas da disciplina Eletrônica analógica que reúne conceitos de eletrônica, circuitos elétricos e atuadores. Trata-se de uma proposta de estudo dos amplificadores de classe B que envolve aplicação de circuitos elétricos equivalentes de transdutores piezelétricos.

Nas bibliografias clássicas utilizadas nas disciplinas nas quais se estuda eletrônica analógica (BOGART, 2001; BOYLESTAD, 2013; HORENSTEIN, 1996; LALOND; ROSS, 1999; MALVINO, 1987; RODEN; CARPENTER; WIESERMAN, 2002; SEDRA; SMITH, 2007), os amplificadores classe B são apresentados na configuração push-pull e analisados considerando-se a carga do amplificador como uma resistência elétrica. Esses estudos apresentam as deduções das expressões das potências consumida na carga, fornecida pela fonte de alimentação e dissipada nos transistores, e, consequentemente, da eficiência.

Os amplificadores de classe B são usados como amplificadores de corrente. Uma aplicação muito comum desses amplificadores é no acionamento de cargas de potências mais elevadas, como os alto-falantes em sistemas de som. Outra possível utilização desses amplificadores é no acionamento de transdutores piezelétricos usados em sistemas de ultrassom de alta potência (WALLENHAUER et al., 2009). Nesses sistemas, muito utilizados na indústria para procedimentos de limpeza, soldagem e corte, o transdutor é excitado na sua frequência de ressonância e converte parte da energia elétrica armazenada em energia mecânica de vibração.

Uma das formas de analisar o comportamento dos transdutores piezelétricos é por meio de circuitos elétricos equivalentes (BALATTO, 2000). Esses circuitos são constituídos por componentes passivos e analisados pelos métodos convencionais estudados na teoria de circuitos elétricos. No entanto, os componentes dos circuitos equivalentes podem sofrer variações devido a efeitos externos decorrentes da operação do transdutor, tais como alterações da carga acústica e da amplitude de vibração, degradação do pré-tensionamento mecânico, entre outros.

As maiores amplitudes de vibração do transdutor piezelétrico são alcançadas na ressonância, quando a saída do amplificador "enxerga" uma carga resistiva. No entanto, devido às variações mencionadas, a carga do amplificador pode passar a apresentar componentes reativas. As componentes reativas afetam o desempenho do amplificador.

Este trabalho apresenta uma proposta de análise de um amplificador classe B que aciona um transdutor piezelétrico e que, portanto, alimentará uma carga que pode apresentar componentes reativas. Esse é o aspecto que promove a contribuição para o ensino, pois trata-se de uma proposta que vai além daquilo que é apresentado convencionalmente nos livros didáticos de eletrônica analógica. Além disso, este trabalho resgata a possibilidade de utilizar a teoria de circuitos elétricos com aplicações práticas na geração de ultrassom ao integrar a ideia de utilizar um circuito elétrico como representação de um dispositivo eletromecânico.

$\mathrm{Na}$ seção de fundamentação teórica são apresentadas informações sobre os circuitos elétricos equivalentes e sobre a análise de circuitos que leva aos cálculos das potências elétricas desenvolvidas nos amplificadores classe B. Na seção de metodologia são descritos 
os procedimentos para determinar a carga do amplificador e suas variações decorrentes do processo de utilização do transdutor e as simulações computacionais que conduzem aos resultados deste trabalho. $\mathrm{Na}$ seção de resultados e discussões são apresentados os resultados dos procedimentos realizados e analisadas as particularidades decorrentes da variação da carga em relação ao desempenho do amplificador. Finalmente, na seção de conclusão são apresentadas as possibilidades que tornam viável o emprego desta proposta como atividade de ensino.

\section{FUNDAMENTAÇÃO TEÓRICA}

\section{Circuitos elétricos equivalentes de transdutores piezelétricos}

Entre os circuitos elétricos equivalentes para dispositivos piezelétricos o circuito de Butterworth - Van Dyke (BVD) (BERLINCOURT; CURRAN; JAFFE，1964; ARNAU, 2008) é um dos mais comuns e adequado para representar os transdutores de ultrassom de alta potência. Na Figura 1 é apresentada a representação esquemática do circuito BVD. O modelo é composto por dois ramos: um elétrico, formado pela capacitância $\mathrm{C} 0$, intrínseca das cerâmicas piezelétricas; outro mecânico, formado por L1, C1 e R1, cujos valores associam-se às propriedades inerciais, elásticas e dissipativas, respectivamente.

Figura 1 - Circuito elétrico equivalente de Butteworth-Van Dyke (BVD)

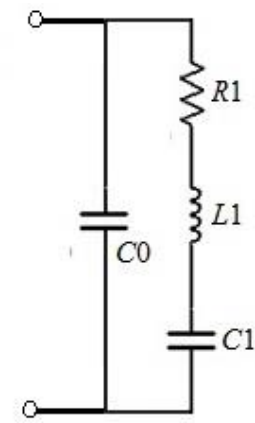

Fonte: elaborada pelos autores.

Na Figura 2 vemos uma curva típica da impedância de um transdutor piezelétrico.
Notamos que os valores mínimos e máximos da impedância correspondem às frequências de ressonância $\left(f_{r}\right)$ e antirressonância $\left(f_{a}\right)$ do circuito. Também é verificado que as defasagens assumem características capacitivas, em frequências inferiores à ressonância e superiores à antirressonância, e indutiva, entre a ressonância e a antirressonância.

Figura 2 - Módulo da impedância elétrica (traço vermelho) e fase (traço azul) de um dispositivo piezelétrico no entorno da ressonância

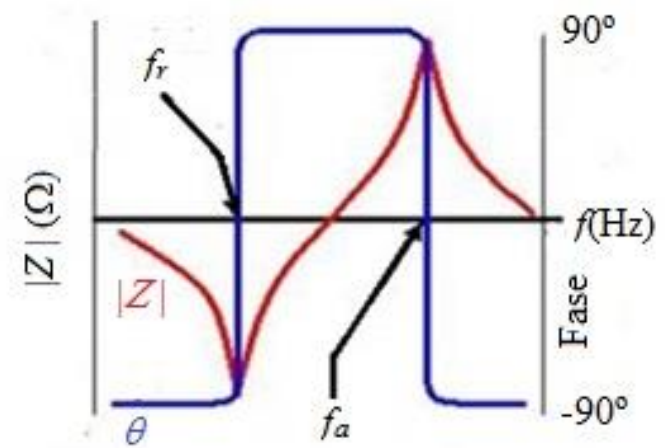

Fonte: elaborada pelos autores.

O modelo BVD é adequado para representar os transdutores de ultrassom de alta potência nas proximidades da ressonância. $\mathrm{Na}$ ressonância do ramo mecânico, a impedância elétrica do transdutor alcança um valor mínimo, que corresponde à máxima condição de conversão eletromecânica. No entanto, nessa condição ainda há uma componente reativa produzida por $\mathrm{C} 0$. Essa componente pode ser eliminada associando-se um indutor de sintonia L0 em paralelo com o transdutor que ressoe em $f_{r}$. A Figura 3 mostra o circuito resultante dessa associação e que será empregada como carga do amplificador e denominada TPL0.

A impedância elétrica total de TPL0 é dada pela Equação 1

$$
Z_{L 0}=Z_{R L 0}+j Z_{I L 0}
$$

onde $Z_{R L 0}$ e $Z_{I L 0}$ são as componentes real e imaginária de TPL0 dadas pelas Equações 2 e $3, R_{L 0}$ e $X_{L 0}$ são as componentes real e imaginária do indutor $L 0, Z_{R}$ e $Z_{I}$ são as componentes real e imaginária da impedância do transdutor dadas pelas Equações 4 e 5, $\omega=$ $2 \pi f$ é a frequência angular em $\mathrm{rad} / \mathrm{s}$ e $f$ é a frequência em Hz. 


$$
\begin{gathered}
Z_{R L 0}=\frac{\left(Z_{R}^{2}+Z_{I}^{2}\right) R_{L 0}+Z_{R}\left(R_{L 0}^{2}+X_{L 0}^{2}\right)}{\left(Z_{R}+R_{L 0}\right)^{2}+\left(Z_{I}+X_{L 0}\right)^{2}} \\
Z_{I L 0}=\frac{X_{L 0}\left[Z_{R}^{2}+Z_{I}^{2}+X_{L 0} Z_{I}\right]+Z_{I} R_{L 0}^{2}}{\left(Z_{R}+R_{L 0}\right)^{2}+\left(Z_{I}+X_{L 0}\right)^{2}} \\
Z_{R}=\frac{R 1(C 1)^{2} \omega}{(L 1 C 1 C 0)^{2} \omega^{5}+\left[(R 1 C 0 C 1)^{2}-2 L 1(C 0 C 1(C 0+C 1)] \omega^{3}+(C 0+C 1)^{2} \omega\right.} \\
Z_{I}=\frac{-(L 1 C 1)^{2} C 0 \omega^{4}+\left[L 1 C 1(C 1+2 C 0)-(R 1 C 1)^{2} C 0\right] \omega^{2}-(C 0+C 1)}{(L 1 C 1 C 0)^{2} \omega^{5}+\left[(R 1 C 0 C 1)^{2}-2 L 1(C 0 C 1(C 0+C 1)] \omega^{3}+(C 0+C 1)^{2} \omega\right.}
\end{gathered}
$$

Figura 3 - Diagrama esquemático do circuito equivalente BVD em paralelo com indutor LO (TPL0)

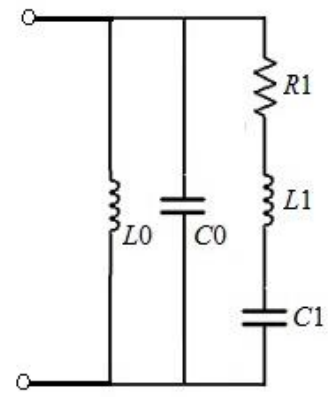

Fonte: elaborada pelos autores.

Portanto, a impedância quando escrita em termos de suas componentes real e imaginária (Equação 1) para uma dada frequência $f$ pode ser convertida em um circuito RL ou RC, série que servirá de carga para o amplificador.

\section{Amplificadores classe B}

O diagrama esquemático do amplificador classe B que iremos estudar é mostrado na Figura 4.

Figura 4 - Diagrama esquemático de um amplificador classe B com transistores bipolares

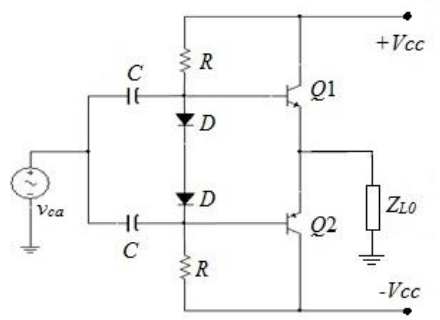

Fonte: elaborada pelos autores.
Nessa configuração de amplificador os transistores bipolares Q1 e Q2 são complementares e estão polarizados na região de corte. Os diodos $\mathrm{D}$ são utilizados para minimizar os problemas de crossover. Teoricamente, esses diodos possuem características iguais às junções base-emissor dos transistores e, desde que se admita que as correntes de base sejam desprezíveis, estabelece-se uma configuração de espelho de corrente, isto é, a corrente de polarização pela malha dos coletores dos transistores é a mesma da malha formada pelos Rs e Ds. Isso significa, portanto, que os transistores devem ser polarizados no limiar entre as regiões de corte e ativa. Outros detalhes sobre a operação desse tipo de amplificador são encontrados nas referências bibliográficas (BOGART, 2001; BOYLESTAD, 2013; HORENSTEIN, 1996; LALOND; ROSS, 1999; MALVINO, 1987; RODEN; CARPENTER; WIESERMAN, 2002; SEDRA; SMITH, 2007) e não serão repetidos aqui.

As Equações 6 a 9 apresentam as potências médias consumidas pela carga, fornecidas pela fonte de alimentação e dissipadas nos transistores, e a eficiência do amplificador quando a carga do amplificador é dada por $z L(\omega)$. A dedução dessas equações pode ser encontrada em Bortoni (1999). 


$$
\begin{gathered}
P_{L}(\omega)=\frac{V_{L}^{2}}{2}\left|Z_{L 0}(\omega)\right| \cos \varphi(\omega) \\
P_{S}(\omega)=\frac{2}{\pi} \frac{V_{L}}{\left|Z_{L 0}(\omega)\right|} V_{L \max }\left(1+\frac{V_{C E s a t}}{V_{L \max }}\right) \\
P_{Q}(\omega)=\frac{2}{\pi} \frac{V_{L}}{\left|Z_{L 0}(\omega)\right|} V_{L \max }\left(1+\frac{V_{C E s a t}}{V_{L \max }}\right)-\frac{V_{L}^{2}}{2}\left|Z_{L 0}(\omega)\right| \cos \varphi(\omega) \\
\eta \%(\omega)=\pi V_{L} /\left[\left(4\left(V_{L(p)}\right)\left(1+\left(\frac{V_{\text {CEsat }}}{V_{L \max }}\right) \cos \varphi(\omega)\right)\right]\right.
\end{gathered}
$$

onde $V_{L}$ é a tensão na carga $Z_{L 0}, V_{L m a x}$ é a tensão máxima em $Z_{L 0}, V_{C E s a t}$ é a tensão de saturação entre coletor e emissor nos transistores e $\varphi$ é o ângulo de fase entre tensão e corrente na carga.

\section{METODOLOGIA}

A metodologia deste trabalho está dividida em duas partes. A primeira delas envolve a determinação do circuito BVD de um transdutor piezelétrico comercial e a consequente definição da carga que será utilizada na saída do amplificador. A segunda parte consiste no cálculo dos parâmetros do amplificador considerando-se a excitação da carga na ressonância e desviada desta. Essas situações permitirão conhecer como o amplificador pode ter seu rendimento modificado quando o transdutor passa por alterações provocadas por efeitos externos.

\section{Determinação da carga do amplificador}

Para este experimento utilizamos um transdutor de ultrassom comercial cuja ressonância é de aproximadamente $25 \mathrm{kHz}$. Este transdutor, montado em nosso laboratório, é formado por duas cerâmicas piezelétricas anulares e peças metálicas dispostas nas extremidades do conjunto. As dimensões das peças e os detalhes da montagem do transdutor são apresentadas em Arnold e Martins (2021). $\mathrm{Na}$ Figura 5 vemos uma fotografia desse transdutor. No furo central das peças constituintes do transdutor há um parafuso que faz um pré-tensionamento mecânico do conjunto. Este pré-tensionamento é necessário para evitar a fratura das cerâmicas no semiciclo trativo (ARNOLD, MÜHLEN, 2001).

Figura 5 - Fotografia do transdutor piezelétrico utilizado nos experimentos

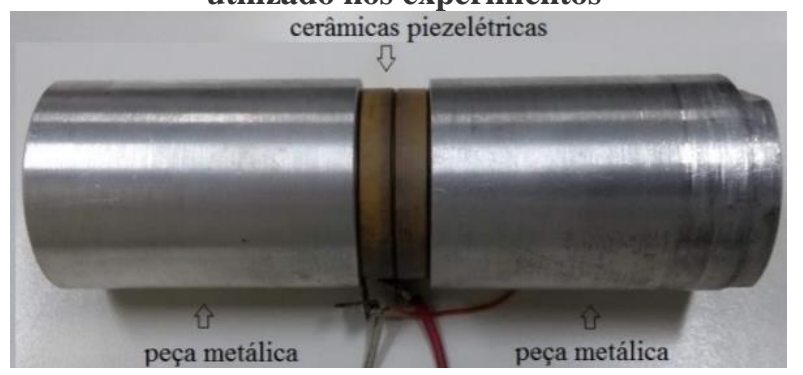

Fonte: elaborada pelos autores.

A curva de impedância em função da frequência desse transdutor pode ser obtida com um analisador de impedâncias HP4194A Agilent. Esse equipamento também pode ser utilizado para determinar os componentes do circuito BVD. Por se tratar de um equipamento de custo elevado que pode não estar disponível em laboratórios de ensino, um método alternativo é utilizar o método da ressonância descrito em Ansi (1987) e Van Raanderat e Setterington (1974). Circuitos mais elaborados que usam o método da ressonância são encontrados em Arnold et al. (2014) e Viana e Arnold (2016). Por meio do método da ressonância é possível determinar as frequências de ressonância e antirressonância e suas respectivas impedâncias. A partir desses valores são calculados os componentes do circuito BVD usando-se a metodologia apresentada por Queiróz, Girão e Serra (2005).

Resultados experimentais encontrados em outras publicações (ARNOLD; MARTINS, 2021; KAUCZOR; FROHLEKE, 2004; SHUYU, 2005; VERRATI; ARNOLD, 2020) 
mostram que o aumento das cargas acústicas e a degradação do pré-tensionamento mecânico deslocam a ressonância do transdutor para valores menores e aumentam o valor do módulo da impedância nessas frequências. Para emular tal comportamento, conforme sugestão de Kauczor e Frohleke (2004), aumentamos os valores de R1 e C1, o que afetará a carga do amplificador em relação aos valores definidos nas condições originais de operação, isto é, com máximo desempenho.

Convém ressaltar que os transdutores que estamos referenciando possuem fator Q elevado. Portanto, pequenas alterações em suas propriedades físicas e, consequentemente, nos valores dos componentes do circuito BVD produzem sensíveis variações de impedância.

\section{Descrição do amplificador}

O estudo do amplificador classe B utilizado neste trabalho foi feito por simulações computacionais. As potências e a eficiência foram determinadas com as equações 6 a 9. A análise da resposta no tempo foi feita por meio da simulação do circuito no LT Spice XVII.

No circuito da Figura 4, os transistores 2N2222 (NPN) e 2N2907 (PNP) são complementares, os diodos D são $1 \mathrm{~N} 4148$, os capacitores C são de acoplamento com capacitância de $1000 \mu \mathrm{F}$, os resistores R são de $5 \mathrm{k} \Omega$, a fonte de alimentação é simétrica de \pm 12 $\mathrm{V}$ e a fonte de sinal de entrada produz sinais senoidais de $12 \mathrm{~V}$ de amplitude e frequência fixa igual a ressonância natural de TPL0. A tensão de saturação (coletor e emissor) dos transistores foi adotada como $V_{C E s a t}=0,1 \mathrm{~V}$. As Equações 6 a 9 foram utilizadas para o cálculo das potências e eficiência do amplificador.

\section{RESULTADOS E DISCUSSÕES}

Os valores dos componentes do circuito BVD, cuja ressonância ocorre em $25367 \mathrm{~Hz}$, são R1 $=43,05 \Omega, \mathrm{L} 1=109,48 \mathrm{mH}, \mathrm{C} 1=$ $359,54 \mathrm{pF}$ e $\mathrm{C} 0=3,57 \mathrm{nF}$. Foi incluído um indutor de sintonia em paralelo com o transdutor para neutralizar a capacitância intrínseca. Esse indutor possui indutância (L0) de $11,14 \mathrm{mH}$ e resistência interna (R0) de
$206,82 \Omega$. Esses valores foram escolhidos por serem o mais próximo do calculado que dispúnhamos em nosso laboratório.

$\mathrm{Na}$ Figura 6 é mostrada a curva de impedância em função da frequência do transdutor utilizado no experimento e de TPLO. Notamos no resultado que a inclusão da indutância de sintonia não altera a frequência de ressonância.

A partir dos valores originais do circuito BVD foram simuladas situações com a introdução das variações de R1 (entre 43,05 $\Omega$ e $83,05 \Omega$ ) e C1 (entre $359,54 \mathrm{pF}$ e $361,54 \mathrm{pF}$ ). $\mathrm{Na}$ Tabela 1 são apresentados os valores das impedâncias e fases calculados a partir da Equação 1.

Figura 6 - Módulo da impedância em função da frequência do transdutor piezelétrico (TP), traço vermelho, e de TPLO, traço preto

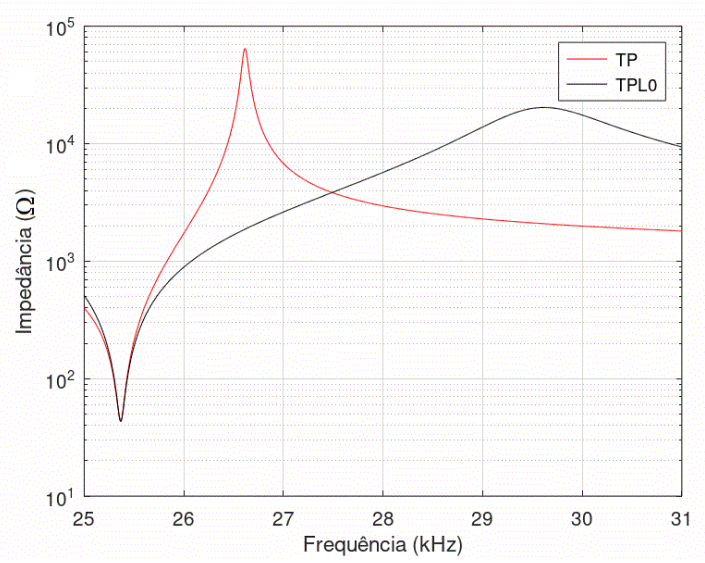

Fonte: elaborada pelos autores. 
Tabela 1 - Valores de impedância / fase e indutância / resistência

\begin{tabular}{|c|c|c|c|c|c|}
\hline & $\mathrm{R} 1 \mathrm{a}=43,05 \Omega$ & $\mathrm{R} 1 \mathrm{~b}=53,05 \Omega$ & $\mathrm{R} 1 \mathrm{c}=63,05 \Omega$ & $\mathrm{R} 1 \mathrm{~d}=73,05 \Omega$ & $\mathrm{R} 1 \mathrm{e}=83,05 \Omega$ \\
\hline $\mathrm{C} 1 \mathrm{a}=$ & $42.98 \Omega /$ & $52.92 \Omega /$ & $62.84 \Omega /$ & $72.76 \Omega /$ & $82.66 \Omega /$ \\
359,54 & $0.55^{\circ}$ & $0.43^{\circ}$ & $0.35^{\circ}$ & $0.29^{\circ}$ & $0.24^{\circ}$ \\
$\mathrm{pF}$ & $2.59 \mu \mathrm{H} /$ & $2.49 \mu \mathrm{H} /$ & $2.41 \mu \mathrm{H} /$ & $2.30 \mu \mathrm{H} /$ & $2.17 \mu \mathrm{H} /$ \\
& $42.98 \Omega$ & $52.92 \Omega$ & $62.84 \Omega$ & $72.36 \Omega$ & $82.66 \Omega$ \\
\hline $\mathrm{C} 1 \mathrm{~b}=$ & $49.54 \Omega /$ & $58.37 \Omega /$ & $67.50 \Omega /$ & $76.82 \Omega /$ & $86.25 \Omega /$ \\
360,04 & $29.67^{\circ}$ & $24.80^{\circ}$ & $21.22^{\circ}$ & $18.51^{\circ}$ & $16.39^{\circ}$ \\
$\mathrm{pF}$ & $153.86 \mu \mathrm{H} /$ & $153.61 \mu \mathrm{H} /$ & $153.29 \mu \mathrm{H} /$ & $153.01 \mu \mathrm{H} /$ & $152.70 \mu \mathrm{H} /$ \\
& $43.04 \Omega$ & $52.99 \Omega$ & $62.92 \Omega$ & $72.85 \Omega$ & $82.74 \Omega$ \\
\hline $\mathrm{C} 1 \mathrm{c}=$ & $65.01 \Omega /$ & $71.95 \Omega /$ & $79.53 \Omega /$ & $87.56 \Omega /$ & $95.94 \Omega /$ \\
360,54 & $48.37^{\circ}$ & $42.39^{\circ}$ & $37.52^{\circ}$ & $33.52^{\circ}$ & $30.21^{\circ}$ \\
$\mathrm{pF}$ & $304.87 \mu \mathrm{H} /$ & $304.34 \mu \mathrm{H} /$ & $303.90 \mu \mathrm{H} /$ & $303.37 \mu \mathrm{H} /$ & $302.88 \mu \mathrm{H} /$ \\
& $43.19 \Omega$ & $53.14 \Omega$ & $63.08 \Omega$ & $73,00 \Omega$ & $82.91 \Omega$ \\
\hline $\mathrm{C} 1 \mathrm{~d}=$ & $84.58 \Omega /$ & $90.01 \Omega /$ & $96.16 \Omega /$ & $102.89 \Omega /$ & $110.10 \Omega /$ \\
361,04 & $59.12^{\circ}$ & $53.64^{\circ}$ & $48.83^{\circ}$ & $44.62^{\circ}$ & $40.95^{\circ}$ \\
$\mathrm{pF}$ & $455.44 \mu \mathrm{H} /$ & $454.78 \mu \mathrm{H} /$ & $454.15 \mu \mathrm{H} /$ & $453.43 \mu \mathrm{H} /$ & $452.74 \mu \mathrm{H} /$ \\
& $43.41 \Omega$ & $53.36 \Omega$ & $63.30 \Omega$ & $73.23 \Omega$ & $83.16 \Omega$ \\
\hline $\mathrm{C} 1 \mathrm{e}=$ & $105.98 \Omega /$ & $110.34 \Omega /$ & $115.39 \Omega /$ & $121.04 \Omega /$ & $127.20 \Omega /$ \\
360,54 & $65.65^{\circ}$ & $60.90^{\circ}$ & $56.55^{\circ}$ & $52.58^{\circ}$ & $48.99^{\circ}$ \\
$\mathrm{pF}$ & $605.78 \mu \mathrm{H} /$ & $604.90 \mu \mathrm{H} /$ & $604.05 \mu \mathrm{H} /$ & $603.13 \mu \mathrm{H} /$ & $602.22 \mu \mathrm{H} /$ \\
& $43.70 \Omega$ & $53.66 \Omega$ & $63.60 \Omega$ & $73.55 \Omega$ & $83.47 \Omega$ \\
\hline
\end{tabular}

Fonte: elaborada pelos autores.

A Figura 8 apresenta exemplos de três curvas da impedância (módulo e fase) no entorno da ressonância do dispositivo piezelétrico (TPL0) em que foram promovidas variações de $\mathrm{R} 1$ e $\mathrm{C} 1$ (curva $1-\mathrm{R} 1=43,05 \Omega$, $\mathrm{C} 1=359,54 \mathrm{pF}$; curva $2-\mathrm{R} 1=53,05 \Omega, \mathrm{C} 1=$ $360,04 \mathrm{pF}$; curva $3-\mathrm{R} 1=63,05 \Omega, \mathrm{C} 1=360,54$ $\mathrm{pF}$ ). Como podemos verificar, os aumentos de
R1 e C1 deslocam a curva da impedância para a esquerda, reduzindo a ressonância e indicando que a carga do amplificador pode ser representada por um circuito equivalente $\mathrm{RL}$ série. A Tabela 1 também apresenta os valores dos componentes do circuito RL resultante.

Figura 8 - Curvas da impedância (módulo e fase) em função da frequência para três pares de R1 e C1
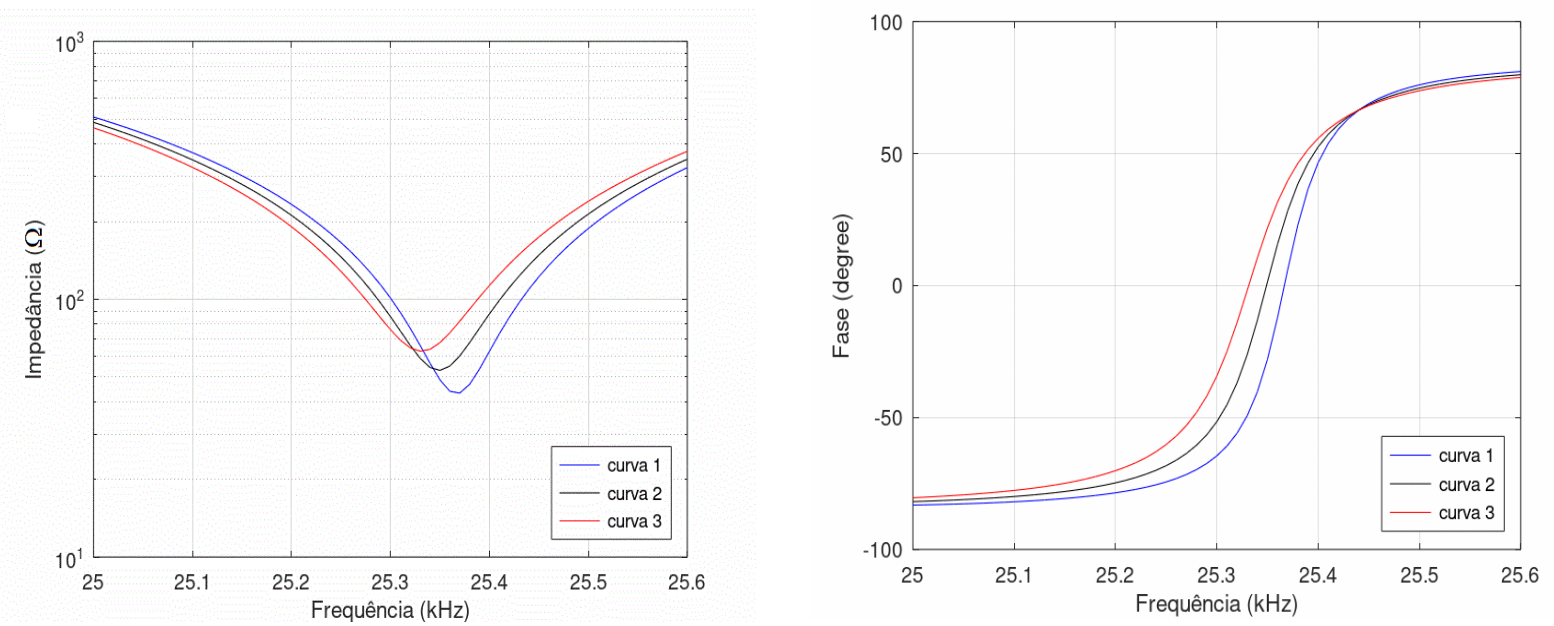

Fonte: elaborada pelos autores. 
Nas Figuras 9 a 11 são apresentadas as potências médias normalizadas do consumo pela carga, do fornecimento da fonte de alimentação e da dissipação nos transistores em função dos valores de R1 e C1. Na Figura 12 é apresentada a potência máxima dissipada nos transistores normalizada. Na Figura 13 é mostrada a eficiência do amplificador.

Nas Figuras 9 e 10 o valor máximo da superfície tridimensional corresponde à ressonância. Verifica-se que quando a ressonância do circuito é desviada, tanto a potência na carga quanto a potência fornecida pela fonte de alimentação apresentam redução. Isso se deve ao aumento da magnitude da impedância da carga resultante. Do ponto de vista prático, significa que as amplitudes de vibração também seriam reduzidas fazendo com que o sistema acústico deixe de operar em sua plenitude. Como essas variações de $\mathrm{R} 1$ e C1 são consequências de efeitos externos ao circuito eletrônico excitador, muitas vezes é empregado um sistema PLL (Phase-Locked Loop) (RAMOS-FERNANDEZ; MONTOYAVITINI; GALLEGO-JUAREZ, 1985; ARNOLD et al., 2015) que corrige a frequência de excitação do transdutor.

Figura 9 - Potência média na carga normalizada $\left(P_{L} / P_{L \max }\right)$ em função de R1 e C1

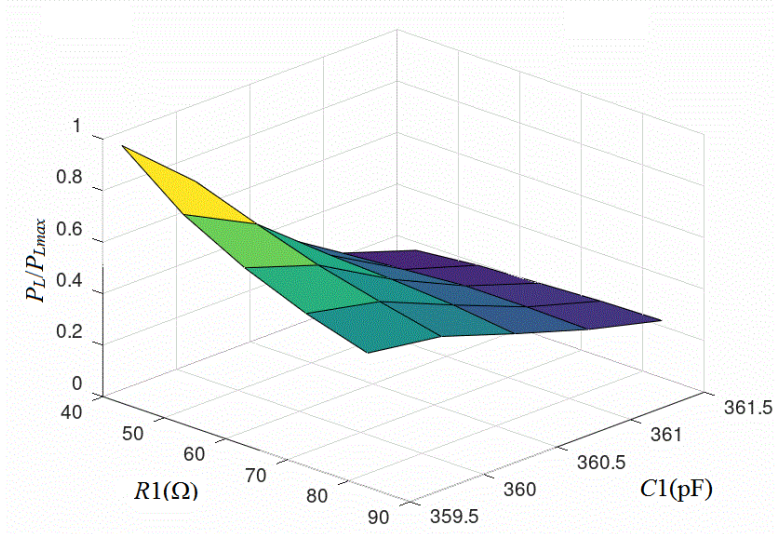

Fonte: elaborada pelos autores.
Figura 10 - Potência média fornecida pela fonte normalizada $\left(P_{s} / P_{L m a x}\right)$ em função de R1 e C1

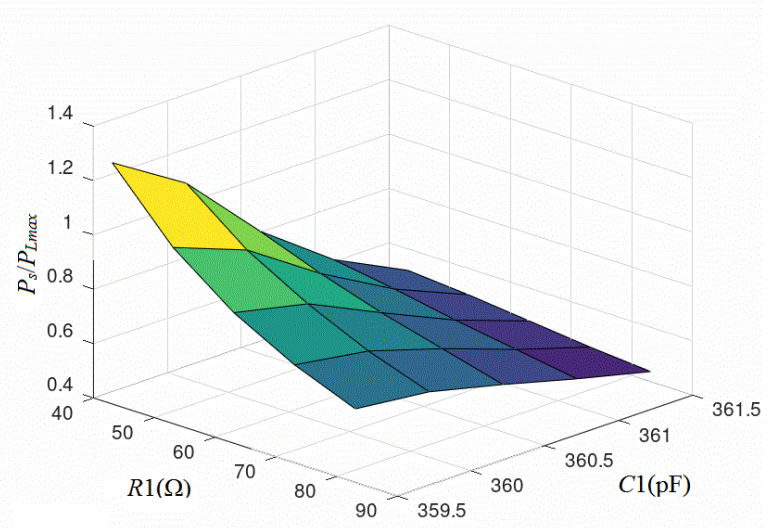

Fonte: elaborada pelos autores.

Figura 11 - Potência média dissipada nos transistores normalizada $\left(P_{Q} / P_{L \max }\right)$ em função de R1 e C1

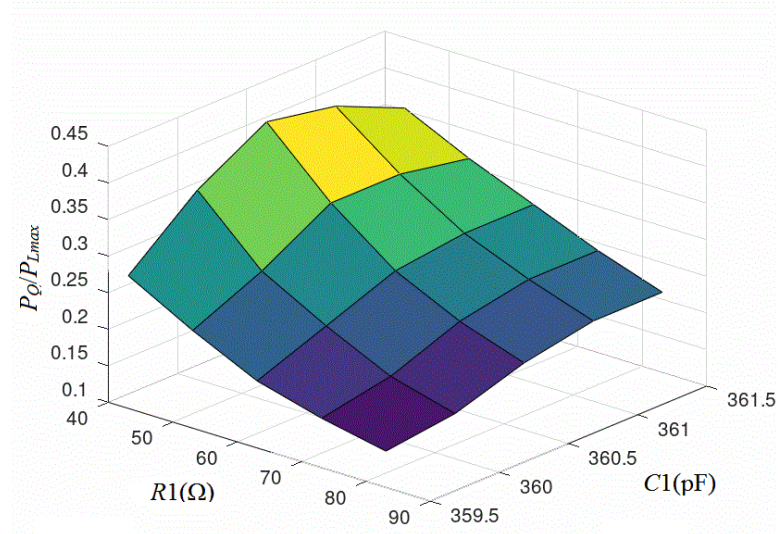

Fonte: elaborada pelos autores.

Figura 12 - Potência máxima dissipada nos transistores normalizada $\left(P_{Q \max } / P_{L \max }\right)$ em função de R1 e C1

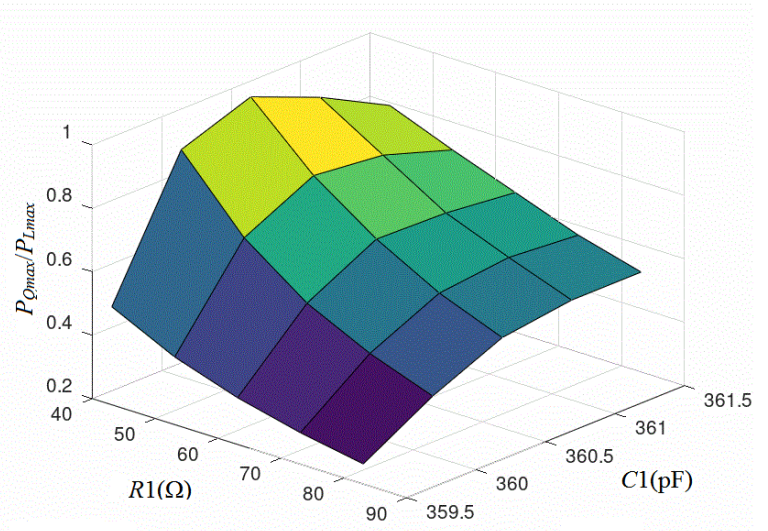

Fonte: elaborada pelos autores. 
Figura 14 - Eficiência do amplificador em função de R1 e C1

As potências de dissipação dos transistores, média e máxima, são mostradas nas Figuras 11 e 12, respectivamente. A presença de componente reativa na carga do amplificador leva a defasagens entre a tensão de saída e a corrente que circula pelos transistores que, por sua vez, está relacionada à potência dissipada pelos transistores. Podemos notar nas Figuras 11 e 12 que os menores níveis de dissipação de potência nos transistores ocorrem para $\mathrm{C} 1=359,5 \mathrm{pF}$, portanto correspondendo às defasagens próximas a $0^{\circ}$.

O aumento da dissipação nos transistores é verificado quando os valores de $\mathrm{C} 1$ estão acima de $360,5 \mathrm{pF}$. Portanto, por meio de uma análise conjunta com os resultados apresentados na Tabela 1, esses valores máximos ocorrem quando as fases estão próximas a $50^{\circ}$ (valor médio de $P_{Q}$ ) e $45^{\circ}$ (valor máximo de $P_{Q}$ ), respectivamente.

Outro ponto a ser destacado no comportamento da curva da potência dissipada nos transistores é o surgimento de uma assimetria decorrente da formação de um pico de potência que se manifesta com o desvio da ressonância, conforme podemos ver no gráfico da Figura 13 para os três casos utilizados como exemplos na Figura 8.

Figura 13 - Curvas da potência dissipada no transistor Q1 em função do tempo para três pares de R1 e C1

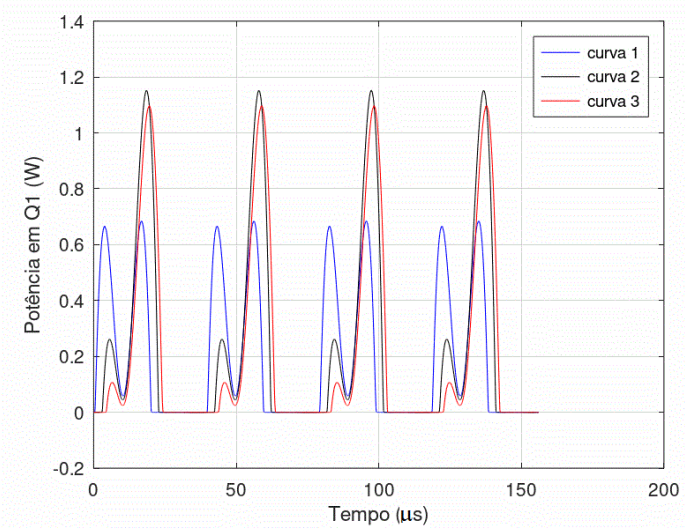

Fonte: elaborada pelos autores.

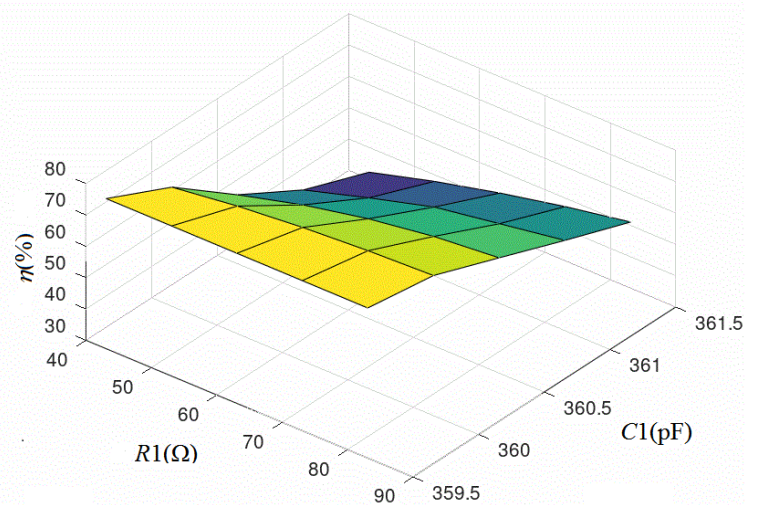

Fonte: elaborada pelos autores.

A eficiência do amplificador é reduzida quanto mais se afasta da ressonância, conforme verificamos na Figura 13. A redução da eficiência e a dissipação nos transistores é mais severa quando os valores de R são menores. A eficiência reduz em cerca de $10 \%$ do seu valor máximo quando as defasagens estão próximas a $25^{\circ}$. Os amplificadores classe B apresentam queda de rendimento mais pronunciada quando o fator $\mathrm{Q}$ dos transdutores é maior.

Usualmente os livros didáticos de eletrônica analógica fazem uma análise do amplificador classe B a partir de variações da amplitude da tensão de entrada. Neste caso estamos interessados no equilíbrio de potências do circuito para atestar a sua eficiência e as condições limites em que ele pode operar satisfatoriamente.

\section{CONCLUSÕES}

Este trabalho ilustra uma aplicação de transdutores piezelétricos, circuitos elétricos equivalentes e eletrônica analógica a qual pode ser utilizada como atividade integradora de disciplinas de cursos de engenharia. Nessa proposta o aluno é instigado a conhecer as possibilidades de utilizar um circuito elétrico equivalente para representar um dispositivo eletromecânico. Essa representação auxilia na identificação e relacionamento das condições de operação do dispositivo e sua integração com os circuitos eletrônicos de acionamento. A proposta também estimula o estudante a refletir em relação ao desenvolvimento de projetos de 
circuitos eletrônicos por meio da análise de suas limitações causadas não apenas dos seus componentes, mas também da influência externa sobre os dispositivos atuadores.

A intenção de utilizar cargas reativas em amplificadores classe $B$ não é tratada nas referências didáticas. Portanto, este trabalho promove uma exploração mais abrangente no estudo desse tipo de amplificador, contribuindo para ampliar a visão do aluno em termos de conteúdos de eletrônica analógica e suas aplicações.

Do ponto de vista técnico, os resultados mostram que há limites para uso de amplificadores classe $\mathrm{B}$, sobretudo quando a carga passa a ter componentes reativas significativas. Tal situação deve ser considerada nos projetos desses amplificadores para essa finalidade e, devido ao elevado fator $\mathrm{Q}$ dos transdutores, a necessidade de circuitos corretores de ressonância deve ser uma alternativa a ser considerada.

Por fim, sugere-se que a proposta apresentada possa ser utilizada e desenvolvida para outras classes de amplificadores e outros dispositivos atuadores com intuito de ampliar as possibilidades de projetos de integração com outras disciplinas dos cursos de engenharia.

\section{AGRADECIMENTOS}

Este estudo foi financiado em parte pela Coordenação de Aperfeiçoamento de Pessoal de Nível Superior, Brasil (CAPES) - Código 001.

\section{REFERÊNCIAS}

ANSI/IEEE, Standard on Piezoelectricity, 176, (1987).

ARNAU, A. Piezoelectric Transducers and Applications. Berlin Heidelberg: SpringerVerlag, 2008.

ARNOLD, F. J.; MÜHLEN, S. S. The resonance frequencies on mechanically pre-stressed ultrasonic piezotransducers. Ultrasonics, v. 39, p. 1-5, 2001.

ARNOLD, F. J. et al. Um sistema simples para medição de impedância elétrica de transdutores piezelétricos. Anais... XX CONGRESSO BRASILEIRO DE AUTOMÁTICA, 2014, Belo Horizonte, 2014, p. 1712-1717.

ARNOLD, F. J. et al. A driver for piezoelectric transducers with control of resonance. Physics Procedia, v. 63, p. 114-119, 2015.

ARNOLD, F. J.; MARTINS, P. S. New insights into the mechanical pre-stressing of piezotransducers. Journal of Intelligent Materials, Systems and Structures, v. $32 \mathrm{n}$. 8, p. 867-879, 2021.

BALLATO, A. Modeling piezoelectric and piezomagnetic devices and structures via equivalent networks. IEEE Transactions on Ultrasonics, Ferroelectrics and Frequency Control, v. 48, n. 5, p. 1189-1240, 2001.

BERLINCOURT, D.; CURRAN, D.; JAFFE, H. Piezoelectric and Piezomagnetic Materials and Their Function in Transducers, Physical Acoustics, v. I-A, ch. 3, Edited by W. P. Mason, 1964.

BOGART JR., T. F. Dispositivos e circuitos eletrônicos. São Paulo: Makron Books, 2001.

BORTONI, R. Análise, dimensionamento e avaliação de estágios de potência de amplificadores de áudio classes A, B, AB, G e H. Dissertação (Mestrado) - Universidade Federal de Santa Catarina, Florianópolis: 1999.

BOYLESTAD, R. Dispositivos Eletrônicos. $11^{\text {a }}$ ed. São Paulo: Pearson Prentice Hall, 2013.

DIRETRIZES CURRICULARES NACIONAIS PARA OS CURSOS DE GRADUAÇÃO EM ENGENHARIA, Ministério da Educação, Conselho Nacional de Educação, Câmara de Educação Superior, Resolução nº 2, 24/4/2019.

HORENSTEIN, M. N. Microeletrônica, Circuitos e Dispositivos. Rio de Janeiro: Prentice-Hall do Brasil, 1996.

KAUCSOR, C.; FROHLEKE, N. Inverter topologies for ultrasonic piezoelectric transducers with high mechanical q-factor. Anais... $35^{\text {th }}$ ANNUAL IEEE POWER ELECTRONICS SPECIALISTS CONFERENCE, p. 2736-2741, 2004. 
LALOND, D. E.; ROSS, J. A. Dispositivos e Circuitos Eletrônicos. São Paulo: Makron Books, 1999.

MALVINO, A. Eletrônica. São Paulo: McGrawHill, 1987.

QUEIRÓZ, R.; GIRÃO, P.; SERRA, A. SingleMode Piezoelectric Ultrasonic Transducer Equivalent Circuit Parameter Calculations and Optimization Using Experimental Data, Anais... IMEKO TC4 SYMPOSIUM, Gdynia, Jurata, pp. 468-471, 2005.

RAMOS-FERNANDEZ, A.; MONTOYA-VITINI, F.; GALLEGO-JUAREZ, J. Automatic system for dynamic control of resonance in high power and high $\mathrm{Q}$ ultrasonic transducer. Ultrasonics, v. 23, p. 151-156, 1985.

RODEN, M. S.; CARPENTER, G. L.; WIESERMAN, W. R. Electronic Design. Los Angeles, CA: Discovery Press, 2002.

SEDRA, A. S.; SMITH, K. C. Microeletrônica. $5^{\text {a }}$ ed. São Paulo: Person Prentice-Hall, 2007.
SHUYU, L. Load characterisitics of high power sandwich piezoelectric ultrasonic transducer, Ultrasonics, v. 43, p. 365-373, 2005.

VAN RANDERAAT, S.; SETTERINGTON, R. Piezoelectric Ceramics. 2 ed. London: Mullard Ltd., 1974.

VERRATI, T. G.; ARNOLD, F. J. An experimental study of the effects of the acoustical load on the piezoelectric transducers performance. Ferroelectrics, v. 568, p. 112-122, 2020.

VIANA, J. C.; ARNOLD, F. J. Desenvolvimento de um sistema automatizado para medição de impedância elétrica. Revista Brasileira de Ensino de Física, v. 39, p. e2503, 2016.

WALLENHAUER, C. et al. Efficient class-B analog amplifier for a piezoelectric actuator drive, Mechatronics, v. 19, p. 56-64, 2009.

\section{DADOS BIOGRÁFICOS DOS AUTORES}
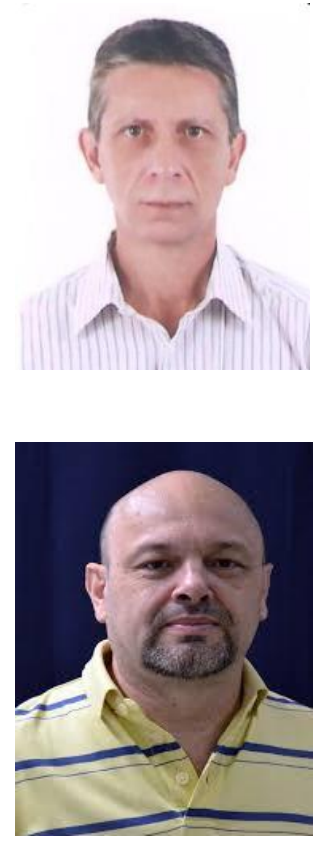

Francisco Antônio Tadheu Ramos - Possui graduação em Engenharia pelo Instituto Superior de Ciências Aplicadas (2009). Tem experiência na área de Engenharia Elétrica, com ênfase em Engenharia Elétrica. Possui especialização em Mecatrônica - Unicamp (2016). Atualmente é mestrando no Programa de Mestrado em Tecnologia da Faculdade de Tecnologia da Universidade Estadual de Campinas.

Francisco José Arnold - Graduado em Física pela Universidade Estadual Paulista (1985), Mestre em Física Aplicada à Medicina e Biologia pela Universidade de São Paulo (1990), Doutor em Engenharia pela Universidade Estadual de Campinas (1995). Atualmente é professor na Faculdade de Tecnologia da Universidade Estadual de Campinas no curso de Engenharia de Telecomunicações no qual ministra as disciplinas Eletrônica Analógica 1 e 2. 